Method. The Urgent and Emergency Care Collaborative (Health Education England) put out a call for funding bids around a number of workforce priority areas. This included upskilling care home staff to reduce admissions. We considered care home staff as those working across supported living schemes, housing with care, residential and nursing homes. Some of these settings exclusively support people with mental health needs.

We obtained a list of accommodation providers across the borough via the Local Authority. As a Community Rehabilitation team we work closely with many of the providers. We also cofacilitate the Hackney Mental Health Supported Accommodation panel and review all funded placements annually. We made contact via email and phonecall and arranged face to face meetings with 11 providers. We asked a standard set of questions about the organisation and training provision. We also asked them to identify gaps in training.

Result. The level of training provided to staff varies vastly across different settings. There was a predominance of e-learning for some providers. Most staff in mental health settings are support worker level which limits the level of training offered/received.

Providers varied greatly in size of project and management structure and this directly impacts on access to training, often as a result of cost.

Providers were able to identify training gaps and were keen to have additional training.

Some common themes emerged - dual diagnosis, psychosis, medication - and some setting specific themes - dementia.

Based on the gaps identified we provided training sessions to a total of $\sim 40$ staff across a number of settings. Content included mental health awareness, crisis signposting and medicines management. All sessions were well received with pre and posttraining questionnaires demonstrating an improvement in knowledge and confidence.

Conclusion. There is potential for knowledge sharing across accommodation settings and for stronger links between accommodation providers and healthcare providers. We plan to explore the possibility of quantitative data on the number of Emergency Department presentations from accommodation settings locally.

\section{Black and minority ethnic groups and forensic mental health}

Donna Arya ${ }^{1 *}$, Charlotte Connolly ${ }^{2}$ and Beth Yeoman ${ }^{2}$

${ }^{1}$ Thornford Park Hospital and ${ }^{2}$ Thornford Park Hospital, Southern Health NHS Foundation Trust

${ }^{*}$ Corresponding author.

doi: 10.1192/bjo.2021.357

Aims. To review the existent literature base regarding Black and Minority Ethnic (BAME) groups care pathway into and experience of care and treatment within secure services. This includes any differences (between BAME and majority ethnic groups) in rates of sentencing, sectioning, length of stay, received treatment and use of restrictive practice. Our overarching aim is to highlight the severe lack of research in this area and the corresponding need for increased research to both consolidate and progress the existing evidence base in order to inform and improve culturally competent service provision.

Background. Research suggests that BAME groups have an increased risk of involuntary psychiatric care, longer-stays within services and higher rates of re-admission. Several explanations have been proposed for this observed disparity, however few of these proposed explanations have provided sufficient or consistent supporting evidence.

Method. A review of both quantitative and qualitative research regarding BAME groups within secure services was conducted. Approximately twenty journal articles, literature reviews and meta-analysis published between 1988 and 2019 were included. The current study should be considered a snapshot and not reflective of the full extent of published literature on the subject. For inclusion, studies should have been conducted in either a forensic mental health setting or a prison and differentiate a minimum of two ethnic groups

Conclusion. Research suggest that BAME individuals continue to experience an increased risk of involuntary psychiatric care, longer stays within secure services and higher rates of re-admission. Whilst many explanations for this disparity have been proposed, few have provided adequate supporting evidence. The ongoing lack of research within this field has led to a limited evidence base from which to inform culturally competent practice. The research which has been conducted has tended to produce inconsistent findings, in part due to the reliance on small scale studies with limited generalisability. Research within this area has been further complicated by varying definitions of culture and ethnicity across studies, leading to some suggestion that the issue of ethnic inequalities and pathways to care, has been misconceptualised. This highlights a critical need for increased research efforts to:

Understand why BAME individuals are at increased risk of involuntary psychiatric care, and how this disproportionate risk can be addressed

Explore potential disparities in the care and treatment of BAME individuals within services and how this might impact upon higher rates of re-admission

Ascertain how best to improve culturally competent service provision.

\section{The introduction of balint groups for core medical trainees - a pilot \\ Itunuayo Ayeni* and Anne Patterson \\ Central and North West London NHS Foundation Trust ${ }^{\star}$ Corresponding author.}

doi: $10.1192 /$ bjo.2021.358

Aims. To introduce and assess the impact of balint groups on core medical trainee (CMT) doctors working within an acute medical trust.

Background. A high rate (80\%) of dissatisfaction and burnout has been reported amongst trainee doctors. This has had a significant impact on recruitment with a large proportion of foundation doctors delaying their application into core specialist training. Of those already in training, up to $50 \%$ have reported taking time, out citing burnout as a cause. Balint groups are a form of reflective practice groups looking at the doctor-patient interaction. For core psychiatric trainees these groups are a mandatory part of their training. Method. We piloted a total of three balint groups over a period of three months amongst CMT doctors based at an acute medical trust in London. A specialty registrar (ST6) in psychiatry facilitated the balint groups. Balint facilitators received supervision from a consultant psychiatrist in psychotherapy. CMT doctors were given questionnaires at the beginning of session one and emerging themes later explored. The questionnaires used were taken from the 'Bristol Trainee-led Balint Group Scheme'.

Result. The pre-questionnaires showed that all CMT doctors surveyed believed psychological factors play an important role on 
patient presentation and recovery. 14/19 (74\%) agreed or strongly agreed that a doctor's reaction to a patient directly influenced care. All doctors agreed or strongly agreed that it was important to reflect on a patient's emotional experience, as it was crucial to their development as a doctor.

CMT doctors found balint groups useful as it provided them a space, which was not routinely offered to discuss challenging cases. Themes that emerged included a lack of support and difficulties maintaining boundaries when treating complex patients. Litigation was a recurring theme with many trainees reporting anxieties and a lack of support. Trainees reported guilt and worries that they were not doing enough for their patients. These themes appeared to have a direct impact on training experience and burnout.

Conclusion. With increasing burnout and dissatisfaction amongst junior doctors, balint groups provide a unique approach to supporting junior doctors within medical specialties. The current pilot has demonstrated that CMT doctors can make use of balint groups in an effective way. We recommend that balint groups should become an integral part of specialist medical training. Psychiatrists can play a central role in supporting the health and well being of medical trainees through balint group facilitation.

The introduction of regular group reflective practice sessions for junior doctors in a critical care setting during the second wave of COVID-19 pandemic a Pilot

Itunuayo $\mathrm{V}$ Ayeni ${ }^{1 \star}$ and Elizabeth Headon ${ }^{2}$

${ }^{1}$ Oxleas NHS Foundation Trust, London and ${ }^{2}$ Lewisham and Greenwich NHS Foundation Trust

${ }^{*}$ Corresponding author.

doi: 10.1192/bjo.2021.359

Aims. Reflective practice is a core component of undergraduate as well as post graduate training. Reflective practice provides an opportunity for individuals to learn through their experience as well as gaining insight into themselves and their practice. If completed effectively, it has been shown to reduce stress and improve mental well-being. Our aim therefore was to provide regular group reflective practice sessions with the aim of supporting junior doctor's mental wellbeing during the second wave of the COVID-19 pandemic.

Method. Junior doctors within a critical care setting were offered two-weekly group reflective practice sessions focusing on 'difficult or challenging cases and encounters.' The sessions were offered to all junior and middle grade doctors within a critical care department in a small district general hospital. Consultants were also able to attend. The groups were facilitated by a consultant liaison psychiatrist and an accredited balint group leader. Critical care doctors were provided a feedback questionnaire assessing the impact of the sessions and the levels of stress and burnout. The themes emerging from the sessions were also explored.

Result. A total of six reflective practice sessions were offered during a three-month period. A total of four reflective practice sessions were completed; two sessions were cancelled due to high workload on the department. Each session lasted approximately 50mins. On average a total of 3-4 junior doctors attended each session. The sessions were conducted face to face in a socially distanced manner and with all participants wearing face masks. The sessions were predominately attended by foundation doctors and SHOs. There was occasional attendance by middle grades and a consultant.

The predominant themes that emerged included: guilt, prolonged suffering, desensitisation, support and exhaustion. Despite the challenges associated with the pandemic and lockdown, many of the doctors also acknowledged the benefit of being at work during both waves of the pandemic. There was a sense of collectiveness and group belonging. The group found it beneficial to be able to share their experiences and challenges faced; this was most striking amongst the very junior members of the team.

Questionnaires were also provided to gain additional insight into the wellbeing of the critical care doctors. Worryingly the results highlighted a significant proportion of doctors were experiencing signs of burnout including fatigue (77\%), lack of energy (54\%), cynicism (31\%), frustration and irritability (45\%) and detachment (38\%). Many of the issues highlighted were in response to the demand created by the pandemic and a lack of medical staffing wth $69 \%$ of doctors requesting regular feedback on staffing issues. Conclusion. Burnout and low morale were already highlighted in a significant number of junior doctors prior to the pandemic. COVID-19 has identified a clear need for NHS employers and medical leaders to provide emotional and psychological support to staff. It is vital that we create an open environment where individuals can express their feelings openly without fear that they will be judged. Group reflective practice provides an avenue to build on collectiveness created during both waves of the COVID-19 pandemic. This pilot has demonstrated that if introduced as part of a wellbeing support package, junior doctors within a critical care setting are able to utilise the sessions in an effective and productive manner.

Factor structure of medical students' attitudes towards psychiatry: findings from a nationally representative sample from Sri Lanka

Anuradha Baminiwatta ${ }^{1 *}$, Miyuru Chandradasa ${ }^{2}$, Dileepa Ediriweera ${ }^{3}$ and Shavindra Dias ${ }^{4}$

${ }^{1}$ Department of Psychiatry, Faculty of Medicine, University of Kelaniya; ${ }^{2}$ Department of Psychiatry, Faculty of Medicine, University of Kelaniya, Ragama, Sri Lanka; ${ }^{3}$ Computer Centre, Faculty of Medicine, University of Kelaniya and ${ }^{4}$ Department of Psychiatry, Faculty of Medicine, University of Peradeniya

${ }^{*}$ Corresponding author.

doi: $10.1192 /$ bjo.2021.360

Aims. The aim of this study was to examine the factor structure of attitudes towards psychiatry among medical students by using the 'Attitude towards psychiatry-30' (ATP-30) scale, which is one of the most widely used psychometric tools in assessing medical students' attitudes regarding psychiatry. We also aimed to explore the possible existence of meaningful subscales in the ATP-30 scale.

Method. Secondary data from a survey of 743 final-year medical students from nine medical schools in Sri Lanka were subjected to factor analysis. Models based on empirical evidence were tested with Confirmatory Factor Analysis (CFA) for model fit using Comparative Fit Index (CFI), Tucker Lewis Index (TLI), root mean square error of approximation (RMSEA) and Chi square. To explore the underlying latent structure of the scale, Exploratory Factor Analysis (EFA) with oblique (i.e. Promax) rotation was employed. Horn's parallel analysis and goodness-of-fit statistics for a series of EFA models tested with different numbers of factors were used in determining the number of factors to retain. Items conceptually external to the emerging factors or with factor loadings less than 0.4 were discarded. Gender invariance of the final model was tested by configural, metric and scalar invariance. Internal consistency of subscales was assessed using McDonald's omega $(\omega)$.

Result. Three models based on literature (one-, five-, and eightfactor) were disproved by CFA. EFA revealed a six-factor solution, 